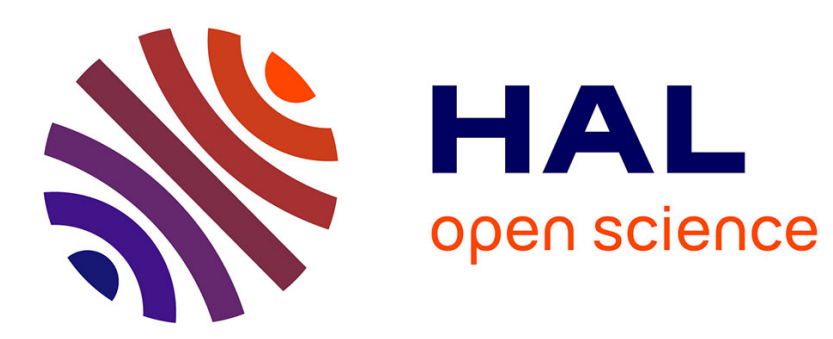

\title{
SERS detection and comprehensive study of p-nitrophenol: towards pesticide sensing
}

Leandro Hostert, Christophe Blanc, Aldo J. G. Zarbin, Eric Anglaret, Elisa S.

Orth

\section{- To cite this version:}

Leandro Hostert, Christophe Blanc, Aldo J. G. Zarbin, Eric Anglaret, Elisa S. Orth. SERS detection and comprehensive study of p-nitrophenol: towards pesticide sensing. New Journal of Chemistry, 2021, 45 (8), pp.3886-3891. 10.1039/d0nj05933a . hal-03194024

\section{HAL Id: hal-03194024 \\ https://hal.science/hal-03194024}

Submitted on 2 Dec 2021

HAL is a multi-disciplinary open access archive for the deposit and dissemination of scientific research documents, whether they are published or not. The documents may come from teaching and research institutions in France or abroad, or from public or private research centers.
L'archive ouverte pluridisciplinaire HAL, est destinée au dépôt et à la diffusion de documents scientifiques de niveau recherche, publiés ou non, émanant des établissements d'enseignement et de recherche français ou étrangers, des laboratoires publics ou privés. 


\title{
SERS DETECTION AND COMPREHENSIVE STUDY OF P-NITROPHENOL: TOWARDS PESTICIDE SENSING
}

\author{
Leandro Hostert ${ }^{\mathrm{a}, \mathrm{b}}$, Christophe Blanc ${ }^{\mathrm{b}}$, Aldo J. G. Zarbin ${ }^{\mathrm{a}}$, Eric Anglaret ${ }^{* b}$ and Elisa S. Orth ${ }^{* a}$ \\ a Department of Chemistry, Universidade Federal do Parana' (UFPR),CEP 81531-980, Curitiba, PR, \\ Brazil.E-mail: elisaorth@ufpr.br
}

b Laboratoire Charles Coulomb, CNRS, Université de Montpellier, Place Eugène Bataillon, 34095 Montpellier Cedex 5, France

\begin{abstract}
Pesticide sensing is an important object of study due to its increasing use worldwide. Herein, we report a SERS study of 4-nitrophenol (PNP), which is product of neutralization processes of various pesticides such as Paraoxon, and can be used as a target molecule for monitoring. PNP is also widely used in the chemical industry and due to its high toxicity is considered a concerning pollutant. The sensing was carried out with a reduced graphene oxide nanocomposite functionalized with cysteamine and $\mathrm{Ag}$ nanoparticles ( $\mathrm{rGOSHAg}$ ), and compared with raw reduced graphene oxide and a commercial SERS substrates (SERStrate ${ }^{\mathrm{TM}}$ ). A mechanistic evaluation was also carried out, focused in the degradation of PNP caused by the different exciting laser lines, evidencing the PNP dimerization in substrates containing Ag NPs (under $532 \mathrm{~nm}$ laser), which has important outcomes for sensing purposes. The nanocomposite rGOSHAg presented the highest sensitivity towards PNP, detecting concentrations as low as 10_6 mol L_1 and with a high potential for field applications and real-time measurements of molecules commonly present in pesticides and industrial contaminants.
\end{abstract}

\section{Introduction}

Pesticide sensing is an important object of study due to its increasing use in different types of plantations. ${ }^{3}$ The construction of devices that perform a fast analysis with high sensitivity and selectivity has become even more crucial to prevent overuse and to increase the rigor of inspection on plantations against the abusive use of pesticides.

In this context some of us have been working towards the destruction of various organophosphatebased pesticides, ${ }^{5}$ but besides degradation there is an interest in obtaining efficient sensors for these compounds. Different sensing methods have been proposed in the literature for pesticides, such as: electrochemical, ${ }^{6-9}$ colorimetric $^{10,11}$ nanomechanical, ${ }^{12}$ and also Surface Enhanced Raman Scattering (SERS). ${ }^{3,11-15}$ SERS is currently wide spreading due to its high specificity, richness of information, easy sample preparation and huge enhancement factors (up to $10^{10}$ ). ${ }^{13}$ The enhanced Raman response is linked to plasmon resonance on nanostructured metals which generates enhanced local electric fields and a concomitant dramatic increase of the Raman intensity. SERS is widely used as a detection tool for different compounds, with use ranging from biosensors for cancer detection, Alzheimer's disease, Parkinson's and glucose, to preservation and identification ofpaintings, or identification of isolated molecules. ${ }^{14-16}$ Some papers have already described the successful use of SERS to detect some chemical agents (chemical weapons/pesticide), such as: mustard gas, dinitrobenzenethiol, malation, ${ }^{17}$ paraquat, ${ }^{18}$ glyphosate, ${ }^{19}$ thiram and thiabendazole, ${ }^{20}$ allowing a new way of detecting harmful molecules.

Herein we propose a method to detect organophosphate (OP) compounds based on monitoring the products of their degradation. Present in many pesticides and also used in chemical weapons, OP presents a high toxicity and can be fatal. ${ }^{21} \mathrm{OP}^{\prime} \mathrm{s}$ degradation and detection are common topics of study due the environmental and health interest. ${ }^{5,22}$ Moreover, the use of pesticide is alarming worldwide 
as well as terrorist episodes involving OP. Recently some of us have designed a nanocomposite based on reduced graphene oxide and silver nanoparticles ( $\mathrm{rGO} / \mathrm{Ag}$ ), in which the material acts both as a nanocatalyst for the degradation and as a SERS substrate to detect the product of the degradation of the triester diethyl 2,4-dinitrophenyl phosphate (DEDNPP), a pesticide simulant. ${ }^{22}$

The present work is centered on a SERS study of 4-nitrophenol (PNP), which is a degradation product of some pesticides such as Paraoxon, Parathion and their derivatives, and can be used as a target molecule to monitor the pesticide degradation (Fig. 1). The sensing was carried out on different substrates: (i) a nanocomposite synthetized in a previous work, $\mathrm{rGOSHAg}^{22}$ which consists of the functionalization of $\mathrm{GO}$ with cysteamine and then the growth of Ag nanoparticles, and for comparison (ii) raw rGO susbtrates and (iii) commercial SERS substrates (SERStratest ${ }^{\mathrm{TM}}$ ). In addition to the SERS sensing of PNP, a mechanistic evaluation was also carried out focused on the degradation of PNP caused by the different exciting laser lines, all this aiming at a construction of a complete SERS substrate for chemical security issues. Furthermore, the sensing of PNP is also of interest in the field of nitrophenols monitoring, which are also widely present in many industrial processes and are highly toxic.

\section{Experimental}

All reagents were used without prior treatment: AgNO3 99.8\% (Sigma-Aldrich), H2O2 30\% (Vetec), H2SO4 98\% (Impex), KMnO4 99\% (Synth), NHS (Sigma-Aldrich), EDC (Sigma-Aldrich), NaBH4 98\% (Acros), NaNO3 99\% (Vetec), graphite (National graphite, Brazil), $\mathrm{HCl} 37 \%$ (impex), ethanol (dynamic), toluene (neon), PNP 80\% (Sigma-Aldrich) and commercial substrates (SERStrates - Silmeco commercial SERS substrates made of foldable silicon pillars plated with gold). All solutions were prepared with deionized water. Experimental details of graphene oxide (GO) synthesis are contained in the SI, the synthesis was previously described by some of us using the adapted Hummers' method. ${ }^{23}$

\section{GO functionalization}

The material's synthesis used in this work was described in a previous work, where GO is functionalized with cysteamine (CA) through the liquid-liquid (L/L) interfacial reaction (toluene and water), named GOSH. To perform the functionalization, coupling reagents N-hydroxysuccinimide (NHS) and 1-ethyl3-(3-dimethylaminopropyl) carbodiimide were used. After the GOSH synthesis, the material was deposited on the substrate of interest. The deposition occurs after transferring the two-phase system to a $25 \mathrm{~mL}$ beaker containing the substrate of interest (glass or silicon). The functionalized film selfassembles at the liquid-liquid interface of the beaker and the substrate is pulled towards the $\mathrm{L} / \mathrm{L}$ interface where the deposition occurs. The deposited material is then dried for $1 \mathrm{~h}$ at $70^{\circ} \mathrm{C}$. More information about reaction times, concentrations or laboratory glassware are contained in the ESI ${ }^{\dagger}$ and in the cited references. ${ }^{22}$

\section{Nanocomposite synthesis}

GOSH was used for the nanocomposite synthesis, also described in a previous work. ${ }^{22}$ For this, two layers of GOSH were deposited on a glass substrate $\left(7.5 \mathrm{~cm}^{2}\right.$ area) followed by its immersion in a closed compartment containing $\mathrm{Ag}+(\mathrm{aq})$, where the system was reduced with $\mathrm{NaBH}_{4}$. The resulting film (rGOSHAg) was then washed with Milli-Q and dried for $1 \mathrm{~h}$ at $70{ }^{\circ} \mathrm{C}$. More information about reaction times, concentrations or laboratory glassware are contained in the ESI+ and in the cited references. ${ }^{22}$

\section{SERS measurements}

Raman measurements were carried out on a Renishaw Invia spectrometer, using three laser lines (532, 633 and $785 \mathrm{~nm}$ and associated edge filters), two gratings $\left(2400 \mathrm{I} \mathrm{mm}^{-1}\right.$ at $532 \mathrm{~nm}$ and $1200 \mathrm{I} \mathrm{mm}^{-1}$ at 633 and $785 \mathrm{~nm}$ ) and a CCD camera detector. Measurements were achieved in a microscope configuration using a $50 x$ long working distance objective. Incident laser powers were fixed to $0.5 \%$ of 
the maximum powers of the lasers, i.e. $156 \mathrm{~mW}$ at $532 \mathrm{~nm}, 26 \mathrm{~mW}$ at $633 \mathrm{~nm}$ and $245 \mathrm{~mW}$ at $785 \mathrm{~nm}$. Measurements at other laser powers are presented in the ESI. ${ }^{\dagger}$

SERS spectra were measured over the spectral range $600-1800 \mathrm{~cm}^{-1}$ with acquisition times of $1 \mathrm{~s}$. Position of the peaks and intensity ratios were determined by deconvolution of the spectra assuming Lorentzian line shapes. The as-fabricated graphene-based nanocomposites deposited over $\mathrm{Si}$ substrates were used as substrates to detect PNP: $20 \mathrm{~mL}$ of PNP solutions $\left(1 \times 10^{-3} \mathrm{~mol} \mathrm{~L}^{-1}\right.$ to $1 \times 10^{-7}$ $\mathrm{mol} \mathrm{L}^{-1}$ ) were dropped over each film and then dried at room temperature for $24 \mathrm{~h}$. The same procedure was performed for comparison on Au NPs assemblies ( $40 \mathrm{~nm}$ commercial suspension from nanoComposix dropped on $\mathrm{Si}$ and dried at room temperature), on raw rGO films and on SERStrates. Besides, PNP over a neat substrate ( $\mathrm{Si}$ ) was also analyzed. Raman mapping images were obtained using the same Raman equipment with different exciting laser lines and powers for each sample with $1 \mathrm{~s}$ accumulation time, and no delay between successive acquisitions.

\section{Results and discussion}

Fig. 2 compares Raman/SERS spectra of PNP dripped on rGO (Fig. 2A) and on the nanocomposite rGOSHAg (Fig. 2B) with two different exciting laser lines ( $532 \mathrm{~nm}$ and $633 \mathrm{~nm}$ ). The two green spectra presented in Fig. 2B correspond to two typical spectra measured on two different areas on the sample. The main bands from PNP are observed at $856 \mathrm{~cm}^{-1}\left(\delta \mathrm{NO}_{2}\right), 1106 \mathrm{~cm}^{-1}(\beta \mathrm{C}-\mathrm{H}), 1335 \mathrm{~cm}^{-1}\left(\nu \mathrm{NO}_{2 \text { sym }}\right)$ and $1575 \mathrm{~cm}^{-1}(\nu \mathrm{C}=\mathrm{C})$. Additional bands are observed for spectra excited at $532 \mathrm{~nm}$ on the nanocomposite at $1148 \mathrm{~cm}^{-1}(\beta \mathrm{C}-\mathrm{H}), 1391 \mathrm{~cm}^{-1}(v \mathrm{NN}+\nu \mathrm{CC}+\nu \mathrm{C}-\mathrm{H})$ and $1435 \mathrm{~cm}^{-1}(\nu \mathrm{NN}+\nu \mathrm{CC}+\beta \mathrm{C}-\mathrm{H}),{ }^{24}$ and are assigned to a dimerization reaction forming the 4,40 -azobis (phenol), that will be discussed in the following. The substrates showed a difference between them which indicates that the presence of $\mathrm{Ag}$ NPs is an important factor for the dimerization reaction. Besides the Ag NPs, the laser line at $532 \mathrm{~nm}$ also plays an important role as illustrated in Fig. 2B: with the $633 \mathrm{~nm}$ laser line, it's not possible to identify any bands attributed to the dimer whereas these bands are systematically observed when measured with the $532 \mathrm{~nm}$ laser line, albeit their relative intensity depends on the probed region, further or closer to a "hot spot" (see two typical spectra with weak and strong intensities in Fig. 2B). This can be explained by a stronger plasmon resonance with silver nanoparticles for the laser line 532 $\mathrm{nm}$ thus favoring local heating and decomposition reactions.

To better understand these results, the same experiment was carried out on two other $\mathrm{Au}$ nanostructured substrates: a thin layer of Au NPs (Fig. 3A) and a SERStrate ${ }^{\mathrm{TM}}$ (Fig. 3B). It is well- known that the plasmon resonance of Au NP shifts to smaller energy (larger wavelength) with respect to $\mathrm{Ag}$ $\mathrm{NP},{ }^{16}$ and an additional redshift is observed in case of aggregated NP. For the two Au-based anisotropic nanostructures we used a thin layer of Au NPs (Fig. 3A) and a SERStrate ${ }^{\mathrm{TM}}$ (Fig. 3B) where the largest Raman intensities are observed for the exciting laser line at $785 \mathrm{~nm}$ (for the SERStrate this optimal plasmon resonance is documented in the provider web site or in the literature). ${ }^{25}$ On the other hand, only the intrinsic signatures of PNP can be detected on these Au-based substrates (the new band at $1303 \mathrm{~cm}^{-1}$ is usually attributable to a ring deformation mode mixed with the nitro group stretching mode).

Photo induced reactions such as dimerization reported here are commonly described in the literature, ${ }^{26}$ mainly in the presence of semiconductors, but some recent work has also described the use of noble metals in this function. ${ }^{27}$ These reactions are usually caused by charge transfer processes between a metal nanoparticle and adsorbed molecules on the metal surface. They are usually described for semiconductors when irradiated with energy greater than the band gap. ${ }^{28}$

An electron-hole pair then can diffuse throughout the sample causing chemical changes in the structure of the adsorbed molecules. In the case of metals, Ag in our case, the process is different, and several hypotheses have been proposed. Nitzan and colleagues have suggested two possible mechanisms to explain the existence of a catalytic activity. The emergence of photoreaction can be attributed to absorption effects enhanced by the plasmon resonance effect, and the second hypothesis 
is linked to the surface heating that occurs on the nano-particles. ${ }^{29}$ Another hypothesis would be a charge transfer from the metallic particles to the LUMO orbitals of the adsorbed molecule. In this case, the Fermi level of the metal would be located between the HOMO and the LUMO of the adsorbed molecule so that if sufficient energy is supplied, the charge transfer process between the components can occur..$^{30}$

Here, it is thus possible to suggest a PNP dimerization mechanism through Ag nanoparticle-catalyzed photoreaction. First, $\mathrm{NO}_{2}$ is reduced to $\mathrm{NHOH}$ by charge transfer from Ag to PNP LUMO orbitals, followed by the condensation reaction between the two groups Ar-NO and Ar- $\mathrm{NHOH}$ to thereby form Ar-NON-Ar, followed by formation of the final product 4,4'-azobis(phenol) (PAP), as illustrated in Fig. 4. The identification of PNP product can be used besides for the dimerization

reaction also for signaling the presence of OP pesticides (ethyl and methyl parathions or Paraoxon) or nitrophenols compounds (which also have high toxicity). ${ }^{31}$

Stability/reproducibility of the SERS signal can be an issue. To address this, we carried out temporal studies by measuring 100 sequential acquisitions (with no interval time between two acquisitions) at two different points. For the first point on the nanocomposite substrate only the intrinsic Raman signatures of PNP were observed (Fig. 5A and B).

No additional peaks were observed at larger times, but the signatures of PNP however become less defined (weaker intensities, broadening and stronger background). The second point (Fig. 5(C and D)) on the other hand exhibits signatures of PNP coexisting with those of the dimer and both the detection of PNP and the dimer are reasonably stable with time.

The two distinct reactions behaviors may be linked to the nanocomposite morphology. As previously described, ${ }^{22}$ the Ag NP have a medium size of $35 \mathrm{~nm}$ and also presented some agglomerated metallic nanostructures over the sample, which can explain the existence of "hot spots" with variable enhancements of the electromagnetic fields depending on the physical parameters of the metal nanostructures. ${ }^{32}$ For comparison, a similar time-evolution study performed on the SERStrate shows a very stable signal over $100 \mathrm{~s}$ (Fig. 6).

After characterization and understanding of the possible responses on the different substrates used in this study, the SERS detection of PNP at various concentrations was compared between rGOSHAg and SERStrate using the optimal excitation lines for each substrate (Fig. 7). A better performance for the graphene nanocomposite (excitation line $532 \mathrm{~nm}$ ) is observed with a possible detection down to $10^{-6}$ mol L-1 of PNP compared to $10^{-5} \mathrm{~mol} \mathrm{~L}^{-1}$ for the commercial substrate (excitation line $785 \mathrm{~nm}$ ). Reproducibility was confirmed by analysis of different points on each sample and also in different films presenting similar results. Another important point that can be highlighted from this experiment is the drop of the Raman signatures of the dimer for rGOSHAg at low PNP concentrations indicating that the dimerization doesn't occur, likely due to limiting kinetics effect since two nearby adsorbed molecules are required for the reaction, which is less likely to happen at low concentrations.

The spectra obtained in Fig. 7 illustrate the high potential of rGOSHAg substrates for PNP sensing, with sensitivity for concentrations down to $106 \mathrm{~mol} \mathrm{L1}$. The material synthesized in this work appears to be even more sensitive (with an excitation line at $532 \mathrm{~nm}$ ) than commercial SERStrates (with an excitation line at $785 \mathrm{~nm}$ ).

In order to compare the results obtained in this work, Table 1 illustrates the minimum concentration detected by other methods described in the literature used for PNP detection. It is worth noting that the vast majority of the techniques for PNP sensor are based on the electrochemical route and the results obtained here are among the best.

The SERS effect for PNP studied here on different materials, allied to the sensing study and the multifunctional behavior of the rGOSHAg as nanocatalyst (illustrated in previous work) ${ }^{22}$ and sensor, illustrates its high potential for a wide range of applications. This work may act as an inspiration for new studies for detecting others toxic compounds/organophosphates, nitroaromatics or pesticides/chemical warfare. More extensive research on this SERS substrate is expected to be conducted, such as modifications of Ag nanostructures to further enhance the SERS signal, use of different metals for NPs or analysis of mixed pesticides in agricultural environments. 


\section{Conclusions}

In summary, we reported a novel strategy to design a multi-functional material to address the chemical security issue involving pesticides and nitrophenol contaminants, based on $\mathrm{rGO}$ nanocomposite functionalized with cysteamine followed by the growth of Ag NPs over the functional groups. The material was prepared as thin films and was evaluated for the first time as a substrate for SERS substrate sensor for PNP.

Results show that the rGOSHAg nanocomposite presents the highest sensitivity towards PNP. In addition to the PNP detection study, this work helped us to confirm the PNP dimerization in substrates containing Ag NPs under $532 \mathrm{~nm}$ laser.

The PNP could be detected at concentrations as low as $10^{-6} \mathrm{~mol} \mathrm{~L}^{-1}$ for rGOSHAg. This work has a high potential, because in addition to presenting substrates with a high catalytic activity against pesticide degradation (already shown in previous works), ${ }^{22}$ it makes it plausible to build multifunctional devices in which both the degradation and sensing of different pesticides would be present. Thus, this study opens an important door for field applications and real-time measurements of molecules commonly present in pesticides or in chemical weapons. Moreover, the field of nitroaromatic detection is also benefited, considering it is widely used in the chemical industry.

\section{Conflicts of interest}

There are no conflicts to declare.

\section{Acknowledgements}

Authors acknowledge the financial support from UFPR, CAPES-COFECUB, CNPq, CAPES, L'OréalUNESCO-ABC, PhosAgro/UNESCO/IUPAC, Fundação Araucária and National Institute of Science and Technology of Carbon Nanomaterials (INCTNanocarbon). This study was financed in part by the Coordenação de Aperfeiçoamento de Pessoal de Nível Superior (CAPES), Brasil, finance code 001. We also acknowledge the financial supports from the ANR program GAELIC ANR-15-CE09-0011-01.

\section{References}

1 M. Wang, B. De Vivo, W. Lu and M. Muniz-Miranda, Appl. Spectrosc., 2014, 68, 784-788.

2 S. Singh, N. Kumar, M. Kumar, Jyoti, A. Agarwal and B. Mizaikoff, Int. J. Chem. Eng., 2017, 313, 283-292.

3 OECD, Agricultural Policy Monitoring and Evaluation, OECD Publishing, 2016.

4 J. Li, D. Kuang, Y. Feng, F. Zhang, Z. Xu and M. Liu, J. Hazard. Mater., 2012, 201-202, 250-259.

5 L. Hostert, R. B. Campos, J. E. S. Fonsaca, V. B. Silva, S. F. Blaskievicz, J. G. L. Ferreira, W. Takarada, N. Naidek, Y. H. Santos, L. L. Q. Nascimento, A. J. G. Zarbin and E. S. Orth, Pure Appl. Chem., 2018, 90, 1593-1603.

6 A. Mulchandani, W. Chen, P. Mulchandani, J. Wang and K. R. Rogers, Biosens. Bioelectron., 2001, 16, 225-230.

7 T. H. V. Kumar and A. K. Sundramoorthy, Anal. Chim. Acta, 2019, 1074, 131-141.

8 R. P. Deo, J. Wang, I. Block, A. Mulchandani, K. A. Joshi, M. Trojanowicz, F. Scholz, W. Chen and Y. Lin, Anal. Chim. Acta, 2005, 530, 185-189.

9 G. Liu and Y. Lin, Anal. Chem., 2005, 77, 5894-5901.

10 M. Liang, K. Fan, Y. Pan, H. Jiang, F. Wang, D. Yang, D. Lu, J. Feng, J. Zhao, L. Yang and X. Yan, Anal. Chem., 2013, 85, 308-312. 
11 N. M. Dissanayake, J. S. Arachchilage, T. A. Samuels and S. O. Obare, Talanta, 2019, 200, 218227.

12 M. Alvarez, A. Calle, J. Tamayo, L. M. Lechuga, A. Abad and A. Montoya, Biosens. Bioelectron., 2003, 18, 649-653.

13 M. Yilmaz, E. Babur, M. Ozdemir, R. L. Gieseking, Y. Dede, U. Tamer, G. C. Schatz, A. Facchetti, H. Usta and G. Demirel, Nat. Mater., 2017, 16, 918-925.

14 D. L. A. d. Faria, M. L. A. Temperini and O. Sala, Quim. Nova, 1999, 22, 541-552.

15 J. Sun, L. Gong, W. Wang, Z. Gong, D. Wang and M. Fan, Luminescence, 2020, 35, 808-820.

16 B. Sharma, R. R. Frontiera, A.-I. Henry, E. Ringe and R. P. Van Duyne, Mater. Today, 2012, 15, 16-25.

17 C. D. L. Albuquerque and R. J. Poppi, Anal. Chim. Acta, 2015, 879, 24-33.

18 H. Fang, X. Zhang, S. J. Zhang, L. Liu, Y. M. Zhao and H. J. Xu, Sens. Actuators, B, 2015, 213, $452-$ 456.

19 R. E. De Go' es, M. Muller and J. L. Fabris, Sensors, 2017, 17, 954.

20 J. Chen, M. Huang, L. Kong and M. Lin, Carbohydr. Polym., 2019, 205, 596-600.

21 K. Kim, O. G. Tsay, D. A. Atwood and D. G. Churchill, Chem. Rev., 2011, 111, 5345-5403.

22 J. E. S. Fonsaca, L. Hostert, E. S. Orth and A. J. G. Zarbin, J. Mater. Chem. A, 2017, 5, 9591-9603.

23 S. H. Domingues, R. V. Salvatierra, M. M. Oliveira and A. J. Zarbin, Chem. Commun., 2011, 47, 2592-2594.

24 B. Bardakçı, T. Kalaycı and N. K. Kınaytü rk, Spectrosc. Lett., 2014, 47, 621-629.

25 D. Cakir, PhD thesis, Université Montpellier, 2017.

26 K. Zhang, N. Xu, M. Jia, R. Li and M. Huang, J. Appl. Phys., 2019, 125, 183101.

27 P. V. Kamat, J. Phys. Chem. C, 2007, 111, 2834-2860.

28 M. Lemos de Souza, D. Pereira dos Santos and P. Corio, RSC Adv., 2018, 8, 28753-28762.

29 A. Nitzan and L. E. Brus, J. Chem. Phys., 1981, 75, 2205-2214.

30 L.-B. Zhao, Y.-F. Huang, X.-M. Liu, J. R. Anema, D.-Y. Wu, B. Ren and Z.-Q. Tian, Phys. Chem. Chem. Phys., 2012, 14, 12919-12929.

31 S. R. Subashchandrabose, M. Megharaj, K. Venkateswarlu and R. Naidu, Environ. Toxicol. Chem., 2012, 31, 1980-1988.

32 M. Rycenga, P. H. C. Camargo, W. Li, C. H. Moran and Y. Xia, J. Phys. Chem. Lett., 2010, 1, 696703.

Figures \& Tables

Figure 1: Generic scheme for the pesticide dephosphorylation, leading to PNP (highlighted in red), the degradation product

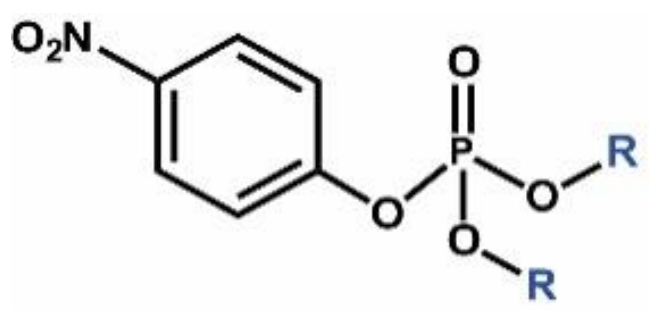

Pesticide/Chemical Warfare

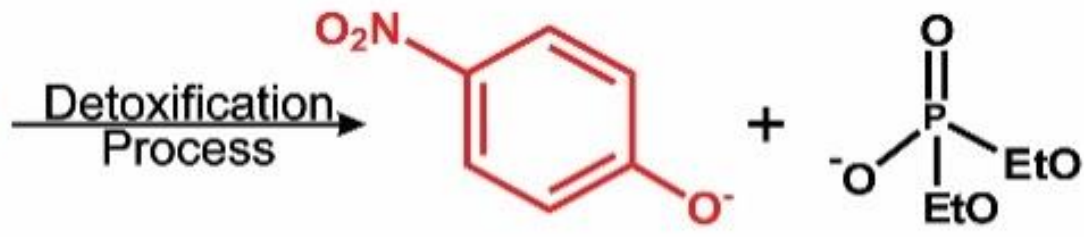

PNP 
Figure 2: Typical Raman Spectra of PNP $\left(1 \times 10^{-3} \mathrm{~mol} \mathrm{~L}^{-1}\right)$ deposited on (A) rGO and (B) rGOSHAg substrates. The peaks labelled in italic are representative of the dimer's band. (B) shows two typical spectra with weak and strong intensities of the dimer's band.
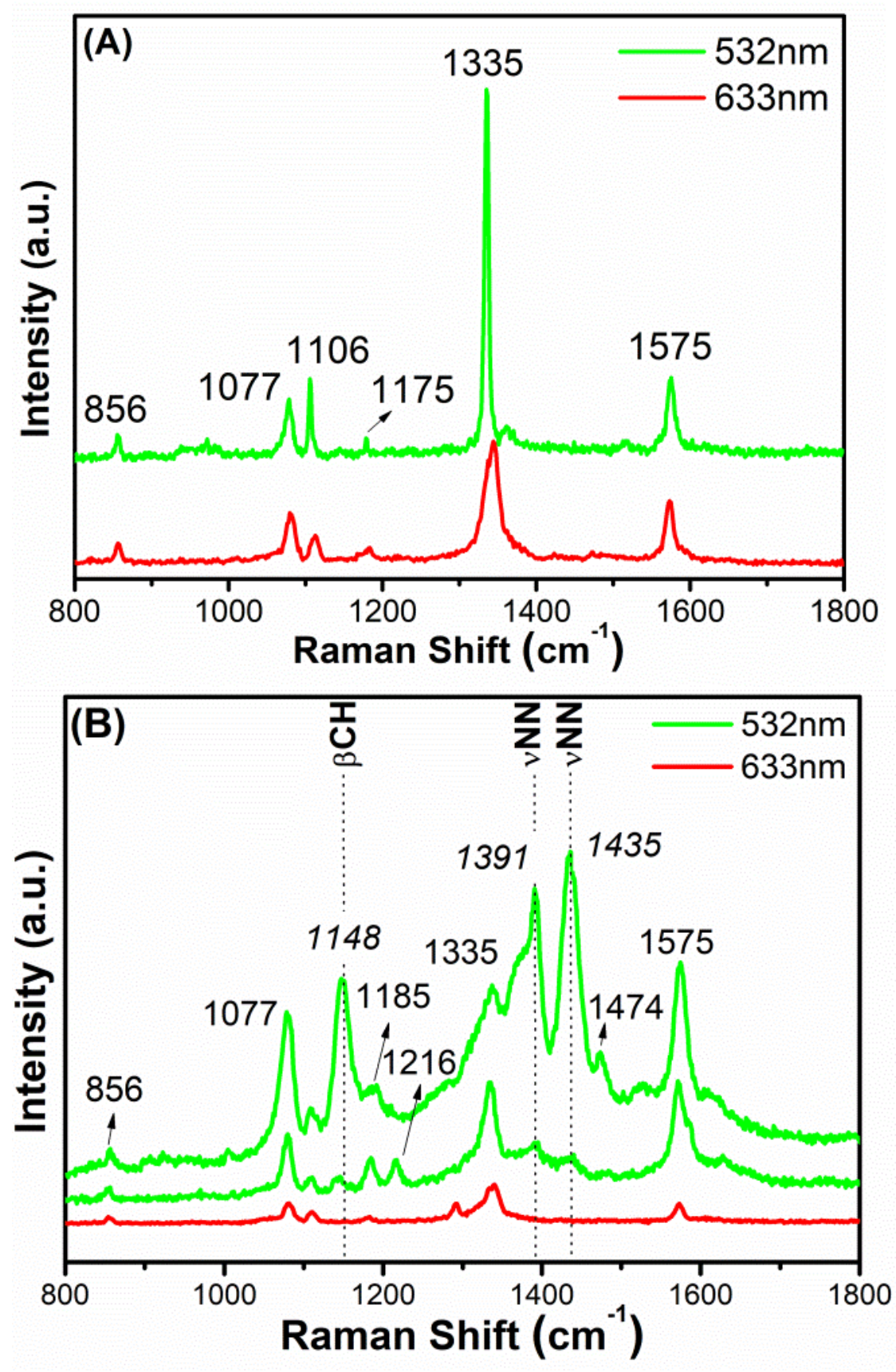
Figure 3: Typical Raman Spectra of PNP $\left(1 \times 10^{-3} \mathrm{~mol} \mathrm{~L}^{-1}\right)$ on (A) Au NPs and (B) SERStrate substrate.
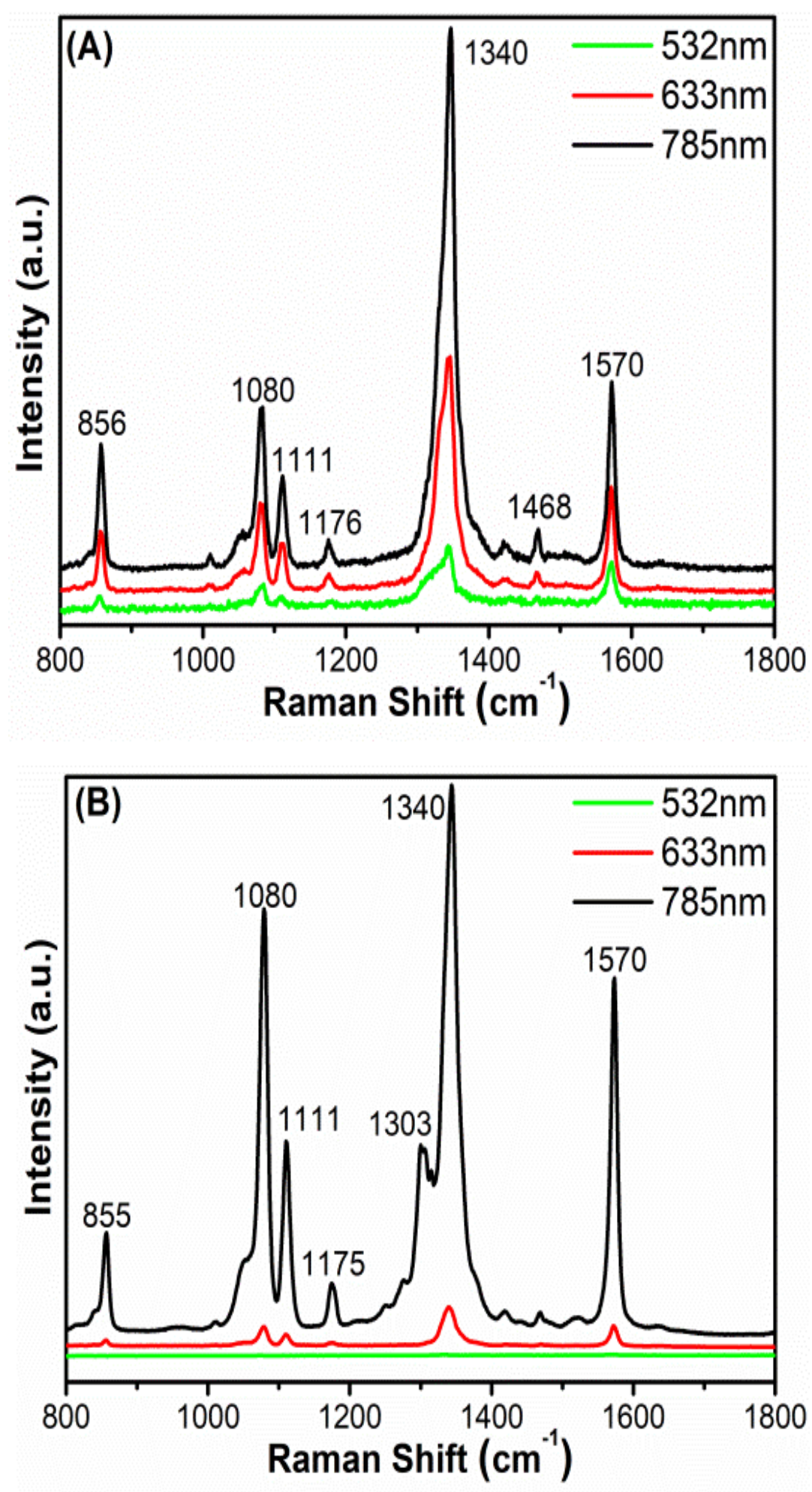
Figure 4: Reactional route for the formation of 4,4'-azobis(phenol) through SERS effect of $\mathrm{Ag}$.
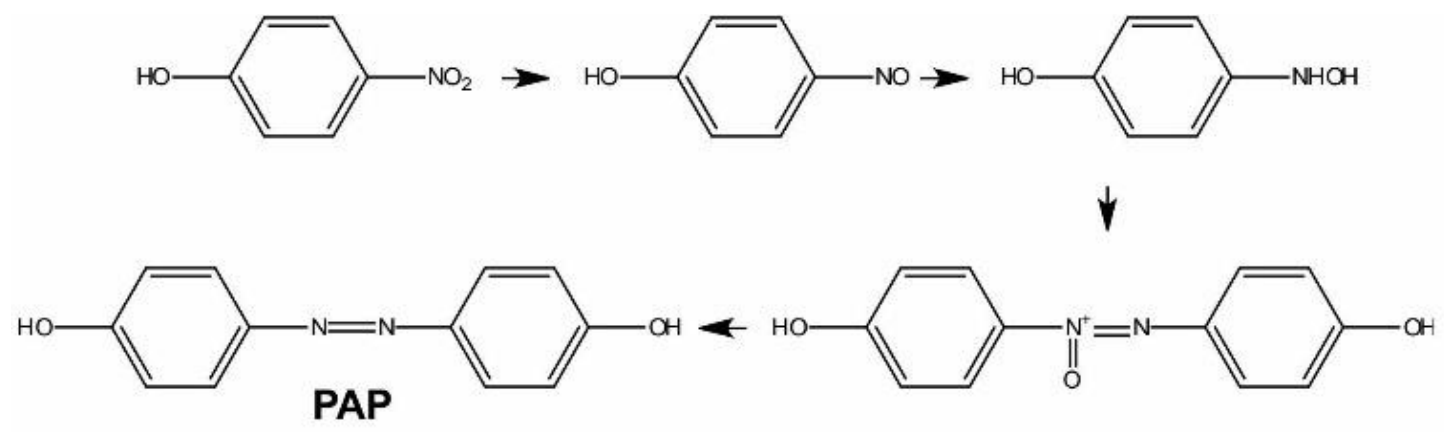

Figure 5: 2 typical sets of temporal evolutions of the Raman spectra of PNP on $\mathrm{rGOSHAg}$ nanocomposites (A-B) at one point and (C-D) at another point of the same sample. Laser line $532 \mathrm{~nm}$, 100 successive spectra of $1 \mathrm{~s}$ without interval time. Left: temporal maps, right: first and last spectra of the series. The peaks labelled in italic are representative of the dimer's band.
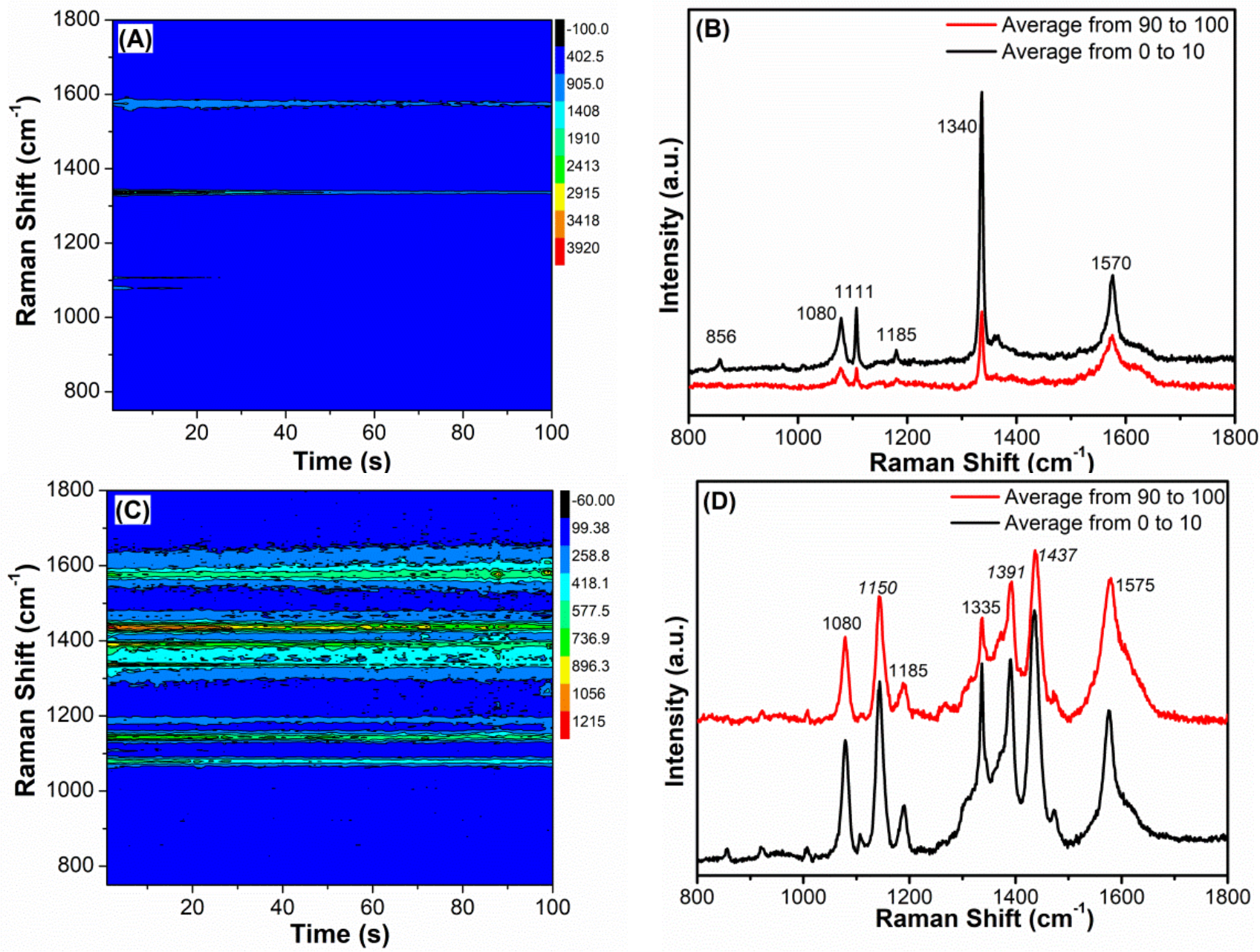
Figure 6: (A) Temporal evolution of the spectrum of PNP dropped on SERStrate. (B) First and last spectra collected from temporal evolution. Exciting line $785 \mathrm{~nm}, 100$ successive spectra of $1 \mathrm{~s}$ without interval time.
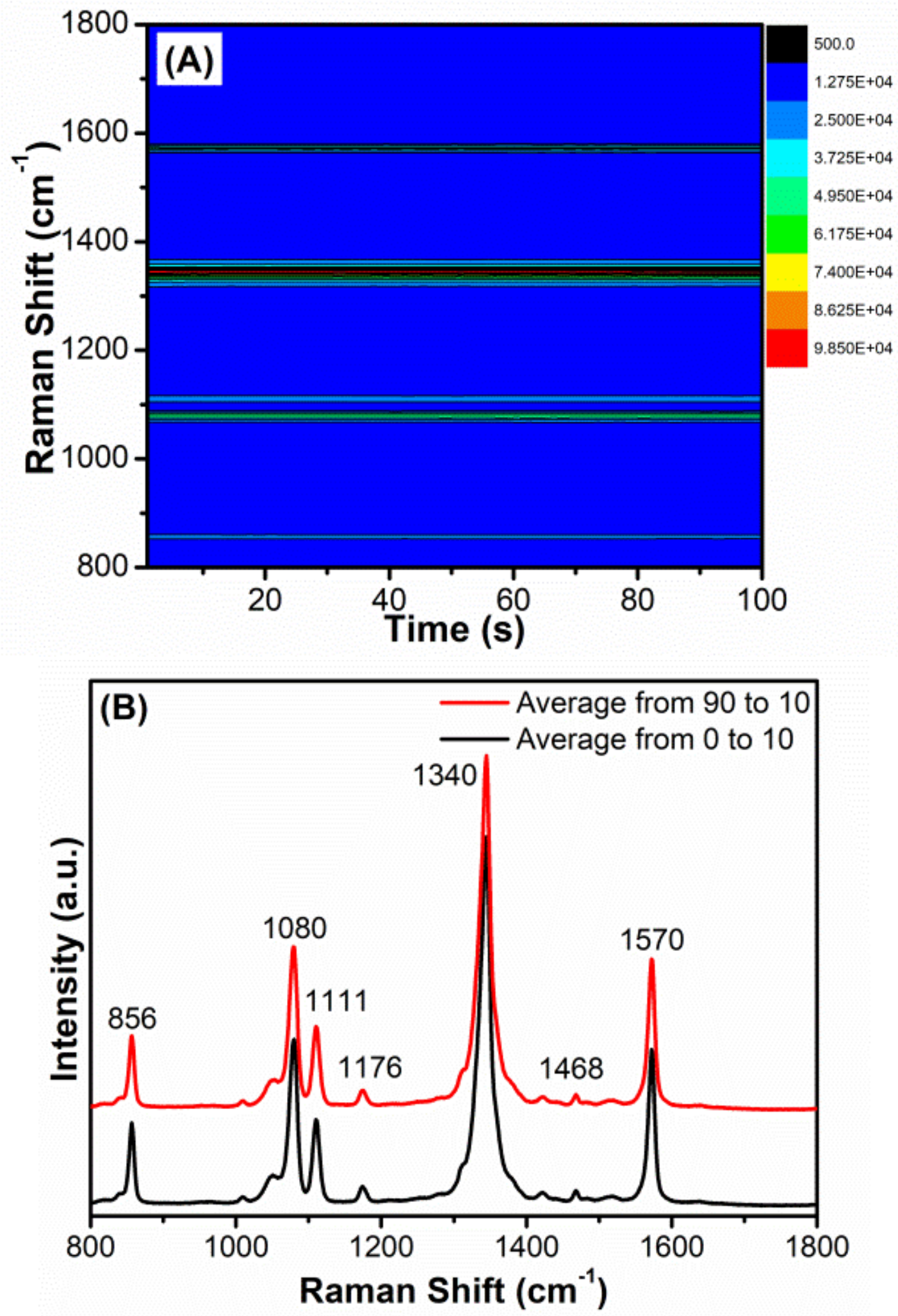
Figure 7: PNP SERS detection study for different concentrations on substrates: (A) rGOSHAg and (B) SERStrate. Measurement conditions: $532 \mathrm{~nm}$ (rGOSHAg) and $785 \mathrm{~nm}$ (SERStrate).
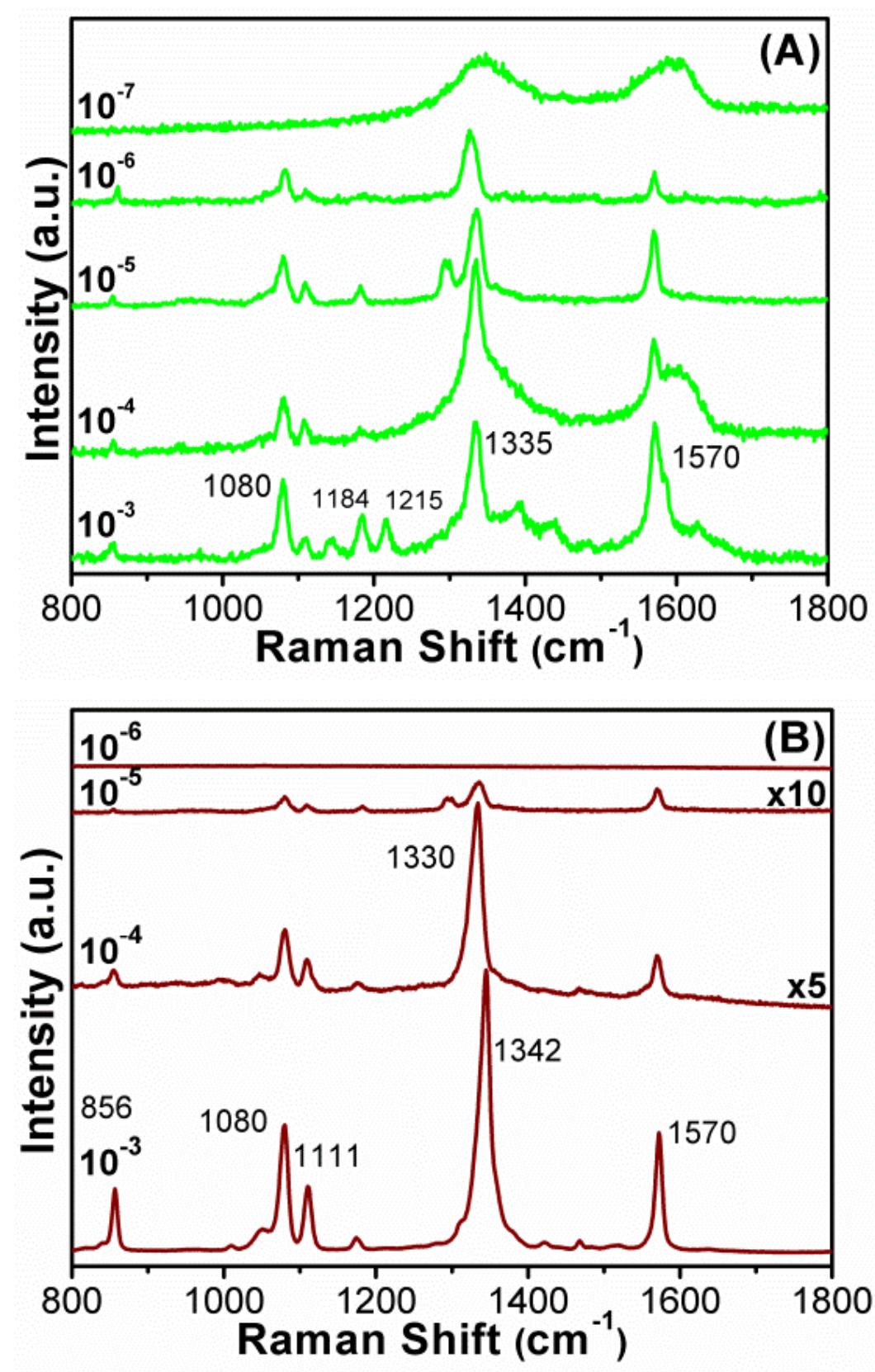
Table 1: Minimum concentration detected for different studies reported in the literature for PNP

\begin{tabular}{|c|c|c|c|}
\hline & Technique & Detection (mol L-1) & Ref. \\
\hline Ag-PDMS & SERS & $10^{-6}$ & 1 \\
\hline SPE/CuO ${ }_{N P s}$ & Electrochemical & $10^{-8}$ & 2 \\
\hline GO/GCE & Electrochemical & $10^{-7}$ & 4 \\
\hline SERSTrate & SERS & $10^{-5}$ & This work \\
\hline rGOSHAg & SERS & $10^{-6}$ & This work \\
\hline
\end{tabular}

